NASA

Contractor Report 195476
Army Research Laboratory Contractor Report ARL-CR-237

\title{
)
}

\section{An Efficient Numerical Procedure for Thermodydrodynamic Analysis of Cavitating Bearings}

D. Vijayaraghavan

University of Toledo

Toledo, Ohio

July 1995

(NASA-CR-195476) AN EFFICIENT

N95-32210

NUMERICAL PRCCEDURE FOR

THERMOHYORODYNAMIC ANALYSIS OF

CAVITATING BEARINGS FInaI

Contractor Report (Toledo Univ.)

P. 26

Prepared for

Lewis Research Center

Under Cooperative Agreement NCC3-291

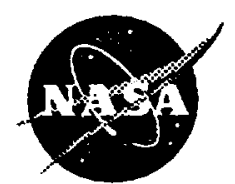

National Aeronautics and Space Administration

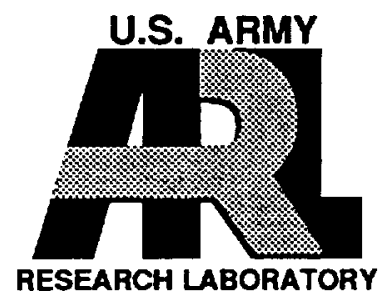




\title{
An Efficient Numerical Procedure for Thermohydrodynamic Analysis of Cavitating Bearings
}

\author{
D. Vijayaraghavan* \\ Dept of Mechanical Engineering \\ University of Toledo \\ Toledo, Ohio 43606
}

\begin{abstract}
In this paper, an efficient and accurate numerical procedure to determine the thermohydrodynamic performance of cavitating bearings is described. This procedure is based on the earlier development of Elrod for lubricating films, in which the properties across the film thickness are determined at Lobatto points and their distributions are expressed by collocated polynomials. The cavitated regions and their boundaries are rigorously treated. Thermal boundary conditions at the surfaces, including heat dissipation through the metal to the ambient, are incorporated. Numerical examples are presented comparing the predictions using this procedure with earlier theoretical predictions and experimental data. With a few points across the film thickness and across the journal and the bearing in the radial direction, the temperature profile is very well predicted.
\end{abstract}

*NASA Resident Research Associate at NASA Lewis Research Center. 


\section{INTRODUCTION}

The increasing trend towards higher speed, higher performance, but smaller size, machinery has pushed the operating conditions of the bearings towards their 'limit design'. Hence, for reliable prediction of the performance of such bearings, a model which accounts for all the operating conditions as closely as possible is becoming increasingly important. Since, the lubricant viscosity strongly depends on temperature, the usual classical assumptions of constant viscosity or effective viscosity become untenable. In many cases, the variation in viscosity can cause a profound impact on performance and it can no longer be considered to have only secondary or perturbation effect.

The evidence of growing interest among researchers in studying the thermal effects is attested by the recent survey by Khonsari (1987) and the workshop on the thermal aspects of fluid film tribology (Pinkus, 1990). The methodology of inclusion of thermal effects spans from approximations by expressing the local temperature distribution by a single value, decoupling of the energy and Reynolds equations and/or assuming the bounding surfaces to be adiabatic, to a full THD model analysis considering heat generation to dissipation through the entire system. Each of these methods has its own merits and limitations. Approximations are aimed at faster solutions while THD models are used for accurate solutions at the cost of computer time.

The temperature variation in a lubricant depends significantly on the lubricant flow rate through the entire bearing. The film rupture and reformation locations, reverse flow and viscous dissipation and heat transfer to metal surfaces in the cavitated region are important in determining the temperature profile. Although most of the studies on thermal effects have included the variations in temperature across the film, generally the cavitation effects have been only superficially considered. Jakobsson-Floberg (1957) - Olsson (1965) have developed boundary conditions (JFO theory), which are based on the conservation of mass principle and the assumption that the fluid flows in the cavitated region in the form of striations extending between the cavitation boundaries and across the film thickness. This model is known to have deficiencies; but nevertheless provides considerable improvement over the earlier more approximate models (Brewe, 1986).

More recently, there have been many contributions in the literature on thermal analysis, which include the cavitation effects. Boncompain et al (1986) used Reynolds boundary conditions for both rupture and reformation, and for the film streamlets in the cavitated region applied the energy equation of the same form as in full film, except that the pressure and transverse velocity were taken as zero. Ott and Paradissiadis (1988) applied JFO boundary conditions to determine the cavitated region and used the same energy equation for both full 
film and cavitated region. However in the energy equation, they used mean value of fluid velocity components, averaged over the film thickness. Han and Paranjpe (1990, 1994a) also applied JFO boundary conditions to determine the cavitated region using the numerical algorithm developed by Elrod (1981). They used a complete energy equation for the entire bearing, with the mass fraction of fluid in the cavitated region included in the energy equation. Later, Paranjpe (1992) and Paranjpe and Han (1994b) extended the analysis for dynamically loaded bearings and non Newtonian effects of the lubricant. Mittwollen and Glienicke (1990) considered mass conservation in their study on the thermal effects on the multi-lobed bearings for turbo machinery.

Although viscosity variations across the film thickness have decisive effect on the bearing performance, viscosity profile in that direction is rather more important than the individual point wise viscosity values. This is because, the coefficients in the hydrodynamic equation are functions of integral of viscosity across the film. By choosing a few points across the field, the location of these points being zeros of the Jacobi polynomial, the profile can be well predicted. Elrod (1991) showed that the conformity using Legendre polynomials at Lobatto points, is much better than with polynomials based on equidistant points. If the order of polynomial is increased, equispaced interpolation may fail to converge while the Legendre polynomial interpolation becomes progressively better. The Lobatto integration considers the end point values also and is a highly accurate scheme. Obviously, lesser the number of points needed across the film, lesser the computational efforts required. Also, when the boundary conditions at film-metal interfaces are in terms of temperature gradients, the unknown boundary temperatures are expressed in terms of all internal Lobatto point temperatures using the polynomial approximation. Hence, the boundary conditions will have direct impact on all the internal points, instead of only the neighboring nodes.

In this paper, a numerical procedure which is both accurate and fast is described. This is achieved by sampling the transverse temperature, flow and velocity values at few chosen points and interlinking them by constructing orthogonal polynomials to determine their profile. Elrod (1991) introduced this basic approach for films. Later, Elrod and Vijayaraghavan (1995) extended the procedure to include cavitation effects also. In this procedure, universal hydrodynamic and energy equations are developed for the entire bearing and mass and energy conservation across the reformation boundary is rigorously implemented. A dual time step procedure is used to solve the THD model for steady state results. Predictions of pressure and temperature profiles using this procedure are compared with various analytical and experimental data available in the open literature. 


\section{NOMENCLATURE}

\begin{tabular}{|c|c|c|c|}
\hline c - & Radial clearance & $\beta$ & - Bulk modulus \\
\hline$c_{p}-$ & Specific heat & $\varepsilon$ & - Eccentricity ratio \\
\hline D - & Journal diameter & $\Phi$ & - Viscous dissipation \\
\hline g & Switch function & $\kappa$ & - Thermal diffusivity \\
\hline h & Film thickness & $\mu$ & - Viscosity \\
\hline$h_{t}-$ & Heat transfer coefficient & $\theta$ & - Partial film content \\
\hline K - & Thermal conductivity & $\rho$ & - Density \\
\hline L & Bearing length & $v$ & - Volume of cell \\
\hline $\mathrm{m}-$ & Mass flux & $\omega$ & - Angular velocity \\
\hline $\mathbf{N}-$ & No. of Internal Lobatto points & $\xi$ & - Fluidity $(1 / \mu)$ \\
\hline$P$ & Legendre polynomial & $\psi$ & - Angular co-ordinate \\
\hline $\mathbf{p}$ & Pressure & $\zeta$ & - Transformed co-ordinate $(2 z / h)$ \\
\hline Q - & Flow rate & & \\
\hline $\mathbf{q}$ & Heat flux & & bscripts \\
\hline $\mathbf{r}$ & Radius & a & - Ambient \\
\hline $\mathrm{T}$ & Temperature & b & - Bearing \\
\hline t & Time & c & - Cavitated region \\
\hline $\mathrm{U}$ & Surface velocity in $\mathrm{x}$ direction & f & - Film \\
\hline $\mathbf{u}$ & Fluid velocity in $\mathrm{x}$ direction & g & - Groove \\
\hline $\mathbf{V}$ & Velocity vector $\left(u e_{x}+v e_{y}\right)$ & $\mathrm{j}$ & - Journal \\
\hline - & Total velocity vector $\left(u e_{x}+v e_{y}+w e_{z}\right)$ & $\mathbf{L}$ & - Lower wall $(\zeta=-1)$ \\
\hline - & Fluid velocity in y direction & $\mathbf{R}$ & - Reference \\
\hline $\mathbf{w}$ & Fluid velocity in $\mathrm{z}$ direction & $\mathbf{s}$ & - Supply \\
\hline $\mathbf{x}$ & Circumferential co-ordinate axis & $\mathrm{U}$ & - Upper wall $(\zeta=+1)$ \\
\hline - & Axial co-ordinate axis & & \\
\hline- & Co-ordinate axis across the $f$ & & \\
\hline
\end{tabular}

Underline - Legendre coefficients

A,B,C,D,E,F,G,R \& S Matrices (see Numerical Formulations) 


\section{THEORETICAL DEVELOPMENT}

The continuity, momentum and energy equations considered in this development are of the following form:

$$
\begin{array}{ll}
\text { Continuity : } & \nabla \cdot v=0 \\
\text { Momentum : } & \frac{\partial}{\partial z}\left(\mu \frac{\partial v}{\partial z}\right)=\nabla p \\
\text { Energy: } & \rho c_{p}\left(\frac{\partial T}{\partial t}+v . \nabla T\right)=K \frac{\partial^{2} \mathrm{~T}}{\partial z^{2}}+\mu\left(\frac{\partial v}{\partial z}\right)^{2}
\end{array}
$$

Basic assumptions are: only viscosity varies with respect to temperature; density and other thermal properties are constant; pressure is constant across the film thickness; Only the film conduction and velocity gradients across the film thickness are significant. All these assumptions are generally accepted for such applications, but does not impose any limitation of this procedure.

The fluidity, $\xi$, which is the reciprocal of viscosity, is used as a variable and expressed in a series of Legendre polynomials, as

$$
\xi(\zeta)=\Sigma \xi_{\mathbf{k}} \mathbf{P}_{\mathbf{k}}(\zeta)
$$

The Legendre coefficients $\xi_{\mathrm{k}}$ are obtained by collocation at Lobatto points. Also, the fractional film gap, $z$, is transformed into $\zeta$ co-ordinates using the relationship $\zeta=z /(h / 2), z$ being measured from the mid film surface. The governing equations for the Legendre coefficients (Elrod, 1991) are

$$
\mathbf{V}=\mathbf{V}_{\mathbf{L}}+\mathbf{A} \int_{-1}^{\zeta} \xi(\varphi) d \varphi+B \int_{-1}^{\zeta} \varphi \xi(\varphi) d \varphi
$$

where

$$
\begin{aligned}
& \mathbf{A}=\frac{\mathbf{V}_{\mathbf{U}}-\mathbf{V}_{\mathbf{L}}-\frac{2}{3} \mathbf{B} \xi_{1}}{2 \xi_{0}} \\
& \mathbf{B}=\left(\frac{\mathrm{h}}{2}\right)^{2} \nabla \mathrm{p} \\
& \frac{\mathrm{m}}{\rho}=\left(\mathbf{V}_{\mathbf{U}}+\mathbf{V}_{\mathbf{L}}\right) \frac{\mathrm{h}}{2}-\frac{\mathrm{h}}{3} \xi_{1} A-\frac{\mathrm{h}}{3}\left(\xi_{0}+\frac{2}{5} \xi_{2}\right) \mathbf{B}
\end{aligned}
$$

The hydrodynamic equation is written as 


$$
12 \frac{\partial h}{\partial t}+6\left(V_{U}+V_{L}\right) \cdot \nabla h-6\left(V_{U}-V_{L}\right) \nabla \cdot\left(\frac{\xi_{1}}{\xi_{0}} h\right)=\nabla \cdot \xi_{p} h^{3} \nabla p
$$

where

$$
\xi_{\mathrm{p}}=\xi_{0}+\frac{2}{5} \xi_{2}-\frac{\xi_{1}^{2}}{3 \xi_{0}}
$$

Here, the expression for $\xi_{p}$ is true, irrespective of number of Lobatto points.

The three dimensional energy equation is written as,

$$
\frac{\partial T}{\partial t}+V \cdot \nabla T+\frac{D \zeta}{D t} \frac{\partial T}{\partial \zeta}=\frac{4 k}{h^{2}} \frac{\partial^{2} T}{\partial \zeta^{2}}+\frac{\Phi}{\rho c_{p}}
$$

where

$$
\frac{D \zeta}{D t}=-\frac{1}{h}\left[(1+\zeta) \frac{\partial h}{\partial t}+\nabla \cdot h \int_{-1}^{\zeta} v d \zeta\right]
$$

\section{Cavitating Bearing}

In the cavitated region, the pressure is taken to be constant at cavitation pressure and the Couette flow is assumed to exist in the form of striations occupying a portion of the volume. The striations extend between the cavitation boundaries and bounding surfaces (see Fig. 1). In a fashion similar to the development of a single 'universal equation' applicable for both full film and cavitated regions for isothermal systems (Elrod and Adams, 1974), the hydrodynamic equation for the present application can be written as,

$$
12 \frac{\partial \theta h}{\partial t}+6\left(V_{U}+V_{L}\right) \cdot \nabla(\theta h)-6\left(V_{U}-V_{L}\right) \nabla \cdot\left(\frac{\xi_{1}}{\xi_{0}} \theta h\right)=\nabla \cdot\left(\xi_{p} h^{3} g \beta \nabla \theta\right)
$$

where

$$
\begin{aligned}
& \theta=\left\{\begin{array}{l}
\rho / \rho_{c} \text { in full film region } \\
\vartheta_{\mathrm{f}} / \vartheta_{\mathrm{t}} \text { in cavitated region }
\end{array}\right. \\
& \beta=\rho \frac{\partial p}{\partial p}=\theta \frac{\partial p}{\partial \theta} \\
& \left.p=p_{c}+\beta(\theta-1) \quad\right\} \text { for } \theta \geq 1
\end{aligned}
$$


Equation (9) has only $\theta$ as independent variable and when solved determines $\theta$ distribution for the entire bearing (thereby pressure distribution) at any instant of time.

According to JFO theory, the liquid film in the cavitated region is confined to the striations. Since, our interest is to determine the film temperature variation in the film, the energy equation at the cavitated region is basically for the striations only. Hence, Eq. (8) is applicable for the striation also. Since length to width ratio of striation will be large, Elrod and Vijayaraghavan (1995) have developed the formulation to evaluate the Lagrangian derivative (Dל/Dt) for the striations, using the short bearing theory which involves some transverse pressure gradient. It has been shown that, within the cavitated region, independent of striation width, we can write the flow gradient term as,

$$
\begin{aligned}
\nabla \cdot \mathrm{h} \int_{-1}^{\zeta} \mathrm{vd} \zeta=\nabla \cdot \int_{-1}^{\zeta} \mathrm{h} \mathbf{V} \mathrm{d} \zeta & =\mathrm{U}_{\mathrm{L}}(1+\zeta) \frac{\partial \mathrm{h}}{\partial \mathrm{x}}+\frac{\mathrm{U}^{-}}{2} \frac{\partial}{\partial \mathrm{x}}\left(\frac{\mathrm{h}}{3 \xi_{0}}\right)\left[\zeta \int_{-1}^{\zeta} \xi \mathrm{d} \zeta-\int_{-1}^{\zeta} \zeta \xi \mathrm{d} \zeta\right] \\
& +3\left(\frac{\partial \mathrm{h}}{\partial \mathrm{t}}+\frac{1}{2} \frac{\partial \mathrm{h}_{\mathrm{c}}}{\partial \mathrm{x}}\right) \int_{-1}^{\zeta}(\zeta-\varphi) \frac{\xi_{\text {int }}(\varphi)}{\xi_{\mathrm{p}}} \mathrm{d} \varphi
\end{aligned}
$$

where

$$
\begin{aligned}
& h_{c}=h\left(U^{+}-U^{-} \frac{\xi_{1}}{3 \xi_{0}}\right) ; U^{+}=U_{U}+U_{L} ; U^{-}=U_{U}-U_{L} \\
& \xi_{\text {int }}=\left[\int_{-1}^{\zeta} \zeta \xi d \zeta-\left(\frac{\xi_{1}}{3 \xi_{0}}\right) \int_{-1}^{\zeta} \xi d \zeta\right]
\end{aligned}
$$

Note that, at $\zeta=1$ this expression reduces to $-2(\partial \mathrm{h} / \partial \mathrm{t})$ and at both upper and lower walls the Lagrangian derivative vanishes, as it should. Also note that there is no transverse temperature variation within the striation and since, the striations are separated from one another, there will not be any convective heat transfer in the axial direction. Since, we are only analyzing the film in the cavitated region, there is no need to include the striation width $(\theta)$ or vary the thermal properties in the cavitated region. However, when heat flux to the boundary surfaces are determined in this region, corresponding striation width should be considered.

Now, we have one governing hydrodynamic and one energy equation applicable to the complete bearing. 


\section{Interface Conditions}

For cavitating bearings, the film reformation interface has to be treated more rigorously. Figure 1 shows the film reformation interface where the striated film abruptly coalesces to form a full film. On the cavitated side of the interface, the film has a Couette flow velocity distribution, while on the full film side, the retarding effect of rising pressure reduces the film's convective capacity. Across the interface, the total mass and energy must be conserved. Conservation of total mass flow is automatically satisfied when the hydrodynamic equation, Eq. (9), is solved. When there is no reverse flow on the film side of the reformation interface, the Lagrangian derivative (D $\zeta / D t)$ adequately describes the fluid motion across the interface and would satisfy the conservation of energy. However, due to adverse pressure gradient in the film side, if a reverse flow occurs in the film side near the stationary wall, then a more detailed consideration of the situation is warranted. Elrod and Vijayaraghavan (1995) have developed a detailed procedure for determining flow pattern and temperature at the film side of the interface for such cases. It is reasonable to assume that across the interface, the temperature associated with a fluid particle does not change much. Thus across the interface, it is hypothesized that

$$
\mathrm{T}=\mathrm{T}(\mathrm{Q})
$$

where

$$
Q(\zeta)=\left\{\begin{array}{l}
\theta \int_{-1}^{\zeta} \mathrm{ud} \zeta \text { in cavitated region } \\
\int_{-1}^{\zeta} \mathrm{ud} \zeta \text { in full film region }
\end{array}\right.
$$

Within the reverse flow region, when $\mathrm{Q}<0$ and $\mathrm{u}<0$, the temperature is determined by the energy equation, from the upstream values. After the velocity becomes positive, but $Q<0$, then for the same flow, $Q$,

$$
\mathrm{T}(\mathrm{Q}, \mathrm{u}>0)=\mathrm{T}(\mathrm{Q}, \mathrm{u}<0)
$$

Beyond this region $(Q>0$ and $u>0)$, the temperature is determined from the flow from cavitated side. For the same flow, $Q$,

$$
[\mathrm{T}(\mathrm{Q})]_{\text {film side }}=[\mathrm{T}(\mathrm{Q})]_{\text {cav }} \text {, side }
$$

In other words, $T(Q)$ is continuous across the reformation interface, but $T(\zeta)$ may be discontinuous.

At the film rupture interface, since $\theta=1$ at both sides of the separation front, no special treatment of the interface is needed. 


\section{Boundary Conditions}

While the cavitation boundary conditions are built into the governing equations, the thermal boundary conditions for the fluid-solid interfaces have to be specified. There are a variety of boundary conditions that can be imposed, based on the total thermal system being considered. When the thermal analysis within the film is of importance, the heat flow to the boundaries of the film are characterized by simplified approximations, namely, constant temperature, adiabatic or no net heat flow boundary. In a full THD model, in addition to considering the heat transfer within the fluid, heat dissipation to the ambient through the boundary surfaces must also be determined. In this way, an actual thermal system is considered for the analysis and hence the predictions will be more realistic.

At the fluid-solid interfaces the temperatures and the fluxes must be continuous. At the film-bearing interface these conditions yield;

$$
\begin{aligned}
& {[\mathrm{T}]_{\mathrm{Z}=\mathrm{h} / 2}=[\mathrm{T}]_{\mathrm{r}}=\mathrm{r}_{\mathrm{bi}}} \\
& \theta_{\mathrm{T}} \mathrm{K}_{\mathrm{f}}\left[\frac{\partial \mathrm{T}}{\partial \mathrm{z}}\right]_{\mathrm{z}=\mathrm{h} / 2}=\mathrm{K}_{\mathrm{b}}\left[\frac{\partial \mathrm{T}}{\partial \mathrm{r}}\right]_{\mathrm{r}=\mathrm{r}_{\mathrm{bi}}}
\end{aligned}
$$

where

$$
\theta_{\mathrm{T}}=\left\{\begin{array}{l}
1 \text { for } \theta \geq 1 \\
\theta \text { for } \theta<1
\end{array}\right.
$$

In the cavitated region, the heat can be transferred to the bearing surface only at the striation surfaces. Hence, the parameter $\theta_{\mathrm{T}}$ is introduced, as $\theta$ indicates the striation width.

The continuity of temperature and flux at the fluid-journal interface is also imposed in similar manner, except that, since the journal is in motion, the cyclic variation of journal surface temperature can be assumed to be constant in the circumferential direction (Dowson et al, 1966). Therefore, the heat flux to the journal surface can be averaged over the circumference. The net heat flux is determined by integrating the film temperature gradient in the following manner (Khonsari and Beaman, 1985).

$$
\begin{aligned}
& {[\mathrm{T}]_{\mathrm{Z}}=-\mathrm{h} / 2=[\mathrm{T}]_{\mathrm{r}}=\mathrm{r}_{\mathrm{j}}} \\
& \frac{\mathrm{K}_{\mathrm{f}}}{2 \pi \mathrm{r}} \int_{0}^{2 \pi \mathrm{r}} \theta_{\mathrm{T}}\left[\frac{\partial \mathrm{T}}{\partial \mathrm{z}}\right]_{\mathrm{z}=-\mathrm{h} / 2} \mathrm{dx}=\mathrm{K}_{\mathrm{j}}\left[\frac{\partial \mathrm{T}}{\partial \mathrm{r}}\right]_{\mathrm{r}=\mathrm{r}_{\mathrm{j}}}
\end{aligned}
$$

The outer surfaces and the lateral surfaces of the bearing transfer heat to the ambient through free convection, depending upon the prevailing heat transfer coefficient. 
In the case of grooved journal bearings, the hot oil carried over mixes with the relatively cooler supply lubricant. Assuming instantaneous mixing at the groove, the temperature of the lubricant leaving the groove is obtained using a simple heat balance. Often, the instantaneous mixing assumption can be questionable in real situations. Heshmat and Pinkus (1986) and Mitsui et al (1983), among others, have tested a number of different bearings and have developed empirical equations to estimate the mixing coefficient used to determine the mixed lubricant temperature. However, for the numerical examples presented here, only simple heat balance is used to determine the oil mixture temperature at the groove.

\section{Heat Conduction Equations}

The heat transfer within the circular bearing shell is governed by the following heat conduction equation, written in cylindrical co-ordinate system,

$$
\frac{\partial T_{b}}{\partial t}=\kappa_{b}\left[\frac{\partial^{2} T_{b}}{\partial r^{2}}+\frac{1}{r} \frac{\partial T_{b}}{\partial r}+\frac{1}{r^{2}} \frac{\partial^{2} T_{b}}{\partial x^{2}}+\frac{\partial^{2} T_{b}}{\partial y^{2}}\right]
$$

In the case of a rotating journal, the journal temperature is considered to be independent of the $x$ direction and the relevant term drops out.

Since, the hydrodynamic and energy equations are set up in unsteady form, the above heat conduction equations are also written in unsteady form. Although in the present paper, only steady state cases are considered, the development of this procedure is aimed at analyzing dynamically loaded applications. Also, for steady state cases, the time derivative term in these governing equations provides time dependent numerical dissipation and has a stabilizing effect on the iteration process. Here, the time step is used as a control parameter for the rate of convergence towards asymptotic steady state conditions

Equations (9), (8), (10) and (14), along with the appropriate boundary conditions, are solved simultaneously to determine the temperature distribution for the whole system. 


\section{NUMERICAL FORMULATIONS}

\section{Matrices}

Elrod (1991) has indicated the procedure to set up the required matrices. For the sake of continuity, the overall approach is mentioned here. It is convenient to set up the fluidity in a vector form and determine the velocity, flow and temperature using matrices. Using matrix notation we can write

$$
\xi\left(\zeta_{k}\right)=\sum_{0}^{N+1} \xi_{n} P_{n}\left(\zeta_{k}\right)=\sum_{0}^{N+1} C_{k n} \xi_{n}
$$

where $P_{n}$ is the Legendre polynomial of order $n, \xi_{n}$ is the Legendre coefficient, $\zeta_{k}$ is the $\zeta$ value at $\mathrm{k}^{\text {th }}$ Lobatto point and $\mathrm{N}$ is the number of internal Lobatto points. Therefore,

$$
\xi=\mathrm{C} \xi \text { and } \xi=\mathrm{C}^{-1} \xi
$$

Similarly, we can also write,

$$
\mathbf{T}=\mathbf{C} \mathbf{T} \quad \text { and } \mathbf{T}=\mathbf{C}^{-1} \mathbf{T}
$$

The velocity profile according to Eq.(5) can be determined from,

$$
\mathbf{V}=\mathbf{V}_{\mathbf{L}}+\mathbf{A} \mathbf{F} \boldsymbol{\xi}+\mathbf{B} \mathbf{G} \boldsymbol{\xi}
$$

The matrices $\mathbf{F}$ and $\mathbf{G}$ can be obtained by evaluating the integrals in Eq.(5) at Lobatto points. Similarly, flow rate obtained by integrating the velocity profile, is computed using

$$
\int_{-1}^{\zeta} \mathbf{V} d \zeta=V_{L}(1+\zeta)+A R \xi+B S \xi
$$

The temperature gradients in the $\zeta$ direction is obtained by successively differentiating Eq.(17), and can also be written in matrix form as,

$$
\frac{\partial \mathrm{T}}{\partial \zeta}=\mathrm{D} \underline{\mathrm{T}} \quad \text { and } \quad \frac{\partial^{2} \mathrm{~T}}{\partial \zeta^{2}}=\mathbf{E} \mathrm{T}
$$

Once the number of internal points is known, the Lobatto point locations, weighting factors and the Legendre polynomials can be determined using standard mathematical handbook; for example refer Abramovitz and Stegun (1965). Then, the matrices C, $\mathbf{C}^{-\mathbf{1}}, \mathbf{D}, \mathbf{E}$, F, G, $\mathbf{R}$ and $\mathbf{S}$ can be easily determined. Note that, these matrices need to be computed only once. 


\section{Cavitation Algorithm}

The 'universal' hydrodynamic equation, Eq.(9), is written in finite-difference formulation using the Elrod cavitation algorithm (Elrod, 1981), appropriately modifying the terms for the present form of the equation. The cavitation algorithm is solved using the two step alternate direction implicit (ADI) method, in which the time step is split into two halves and for each half, $\theta$ values in one co-ordinate direction is implicitly solved. For each sweep, this results in only tridiagonal system of equations to be simultaneously solved. The tridiagonal matrices can be efficiently inverted using Thomas algorithm (refer Anderson et al, 1984). When the process is completed, $\theta$ value distribution is updated for next time step.

\section{Energy Equation}

The energy equation, Eq.(8), is implicitly solved using three dimensional ADI method, developed by Douglas and Gunn (1964) (also refer Anderson et al, 1984). This procedure, which is an extension of Crank-Nicholson scheme to three dimensional form, is second-order accurate in time and space, and is stable. The solution for one time interval is obtained in three steps. At each step, one direction is treated implicitly while the derivatives in other directions are evaluated for known values of temperature. With the present formulation of the energy equation, during the sweep in $\zeta$ direction, ( $\mathrm{N} \times \mathrm{N}$ ) matrix has to be inverted, $\mathrm{N}$ being the number of internal Lobatto points; for the other two directions only tridiagonal matrices have to be inverted.

For the full THD model, the temperature distribution in the fluid, bearing and journal is to be determined. In order to efficiently and simultaneously determine the temperature distribution in the bearing/runner metals and also to effectively couple the boundary conditions at fluid-metal interfaces, Lobatto points are positioned within both the bearing and journal domains. Now, the bearing and journal temperature gradients in $\zeta$ direction can be expressed in matrix form, similar to Eq.(20). Proper care must be taken to index all the Lobatto points, since, different equations, boundary conditions and variables are being used in each domain.

When there are regions of reverse flow in the bearing, computational instabilities could be encountered. The negative velocity hinders the solution of energy equation, which is being treated as initial value problem and marched forward. Also, the convection terms in the energy equation are of first order. Central differencing of these terms sometimes causes numerical instability due to lack of diagonal terms. To alleviate both problems and to preserve numerical stability, one sided upwind differencing is employed for the convection terms. For example, 


$$
\left(\mathrm{u} \frac{\partial \mathrm{T}}{\partial \mathrm{x}}\right)_{\mathrm{i}}=\operatorname{abs}\left(\mathrm{u}_{\mathrm{i}}\right) \frac{\mathrm{T}_{\mathrm{i}}-\mathrm{T}_{\mathrm{i}-\mathrm{igx}}}{\Delta \mathrm{x}} ; \quad \operatorname{igx}=\operatorname{sign}\left(\mathrm{u}_{\mathrm{i}}\right)
$$

Similarly for convection in transverse (y) direction.

\section{Boundary Conditions}

When the boundary surfaces are held at constant temperature, only the temperature distribution at internal Lobatto points need be determined. However, when one or more boundaries have other boundary conditions involving temperature gradients, those boundary temperatures are also unknown. Because of our polynomial approximation procedure, it is possible to express the boundary temperatures and thus the boundary conditions in terms of all the internal Lobatto point temperatures. In the THD model temperature at all the four boundaries, two fluid-metal interfaces, metal-ambient interface and journal center or inside diameter, are unknown. It is still possible to express these boundary conditions in terms of internal Lobatto point temperatures in the form of simultaneous equations. The temperature distribution in the whole system can be solved in implicit manner and from the internal temperatures, boundary temperatures can be determined. Though, this process is somewhat tedious, it is relatively straight forward. For more details, interested readers are referred to Villadsen and Michelsen (1978).

\section{Dual Time Step}

The thermal response of a fluid film is of the order of milliseconds, whereas, the temperature of metal parts takes seconds or minutes to stabilize due to their large thermal capacity. Also, with the numerical procedure point of view, the grid spacing across the film thickness is of the order of microns, while in the metal the grid points are in terms of millimeters apart. When the whole system is solved simultaneously, these differences can cause numerical problems. Khonsari and Beaman (1985) reported that when the intermediate results of the metal conduction equation are directly used as boundary conditions for energy equation, the solution becomes unstable. They used all equations in steady state form and the Reynolds and energy equations were solved several times before moving to heat conduction equation. Paranjpe and Han (1994b) also discussed about the time scales for thermal transients and determined that the time scale for thermal transients for joumal and bearing to be 3 to 4 orders of magnitude greater than those for fluid.

To tackle this problem, a dual time step procedure is utilized here. Smaller time steps (of the order of $10^{-3}-10^{-4} \mathrm{sec}$ ) were used to solve fluid film equations and larger time steps (of 
the order of $10^{0}-10^{-2} \mathrm{sec}$ ) were used in bearing and runner metal domains, and all the equations are solved simultaneously. For steady state problems, the system eventually attains an equilibrium conditions with the surroundings and this dual time scale should not matter. With this dual time march, we are only accelerating the temperature changes in metal compatible with the temperature changes in fluid. This procedure is found to be very stable and relatively faster in convergence. The final solution was also found to be independent of the time step. When the time step used for conduction through the metal is not compatible with the time step used in fluid domain and the solution diverges, then the time steps are adjusted and the analysis is repeated.

\section{Solution Procedure}

When the number of Lobatto points is known, their locations and all the matrices are readily computed. Initially, the whole bearing clearance is assumed to be filled with lubricant and the internal temperatures are set as a function of boundary temperatures. Fluidity values at all points are determined according to the latest available temperatures and the viscositytemperature relationship. The hydrodynamic equation is solved using the cavitation algorithm to determine the $\theta$ distribution and thereby the pressure and mass flow rate. Using the flow vectors $\mathbf{A}$ and $\mathbf{B}$ and other matrices, three dimensional velocities, flows and viscous dissipation at every Lobatto point are determined. Across the film reformation front, the interface conditions as described above are enforced. The energy equation is implicitly solved. After each step, the boundary temperatures are updated. After each time step the convergence in $\theta$ and $T$ are checked and if not adequate, the process is repeated by updating the fluidity values and switch function, $g$, values. In the case of grooved journal bearings, at every time step the mixed lubricant temperature is determined using simple heat balance. The nodes in the groove location are assumed to be at constant supply pressure and at constant mixed lubricant temperature.

The numerical code is written for finite bearings and is vectorized for use on Cray XMP/YMP computers. For the numerical examples discussed in this paper, convergence accuracies of $10^{-6}$ for $\theta$ and $10^{-3}$ for temperature are imposed. However, it is found that the convergence criteria for $\theta$ is more stringent and when the steady state conditions are achieved, the temperature convergence is below $10^{-3}$. Various grid sizes and Lobatto points were tried and it was found that above 41 nodes in the circumferential direction, depending upon L/D, 7 to 11 nodes along half the bearing length in axial direction and 3 Lobatto points, the results are very similar; but the computer time requirement was becoming larger. In order to make use of vectorization of Cray computers, 49 nodes in the circumferential direction, 9-11 nodes axially, 
3 internal Lobatto points across film and journal and 5 internal Lobatto points across bearing radius are chosen. The computer time requirement is primarily dependent on the type of problem being solved. For a typical finite journal bearing, to solve a direct problem with eccentricity specified, with adiabatic boundary conditions, the CPU time on a Cray XMP computer, for the above grid arrangement, was 15-20 seconds and for a typical THD model including journal and bearing, the CPU time requirement was 100-200 seconds.

\section{NUMERICAL EXAMPLES}

With the present procedure, several cases are analyzed and the results are compared with the earlier analytical predictions and experimental data available in literature. The bearing data are shown in Table 1.

Ott and Paradissiadis (1988) and later Han and Paranjpe (1990) analyzed the temperature distribution in an axial grooved journal bearing wherein the journal and oil inlet groove are maintained at constant oil inlet temperature and the bearing surface is adiabatic. The pressure and temperature contours at the axial symmetry plane predicted with just 3 internal Lobatto points are compared with the predictions of Han and Paranjpe (1990), as shown in Figs. 2 and 3, respectively. The trends in these figures are similar to the earlier predictions. Both the present prediction and those of Han and Paranjpe have the same pressure profile with a peak pressure of $18 \mathrm{MPa}$, compared to the value of $14.5 \mathrm{MPa}$ predicted by Ott and Paradissiadis. The temperature contours have some distinct differences. Both Ott and Paradissiadis and Han and Paranjpe predicted the fluid temperatures in the cavitated region to be fairly constant at the same $\zeta$ locations, with the maximum value being $100^{\circ} \mathrm{C}$ and $120^{\circ} \mathrm{C}$ respectively. With the present procedure, as can be seen from Fig. (3), fluid temperatures in the cavitated region increase at the same $\zeta$ locations, with the maximum value being $130^{\circ} \mathrm{C}$ and fluid with lower temperature is closer to the constant temperature moving journal. In the cavitated region, the film thickness increases and the fluid velocity component in the $\zeta$ direction will have to be significant in order to fill in the gap, towards the hotter adiabatic boundary surface. Therefore, it is reasonable to expect the temperature to rise in this region, as predicted by the present procedure. The load capacity, as per the present prediction is $328.4 \mathrm{kN}$ as against $273.2 \mathrm{kN}$ obtained by Ott and Paradissiadis.

Ferron et al (1983) conducted experiments to measure the temperature variations in a journal bearing and compared the data with their theoretical predictions. They determined that due to differential dilatation of the steel journal and bronze bearing, the nominal radial clearance of $145 \mathrm{~mm}$ at $20^{\circ} \mathrm{C}$ became $152 \mathrm{~mm}$ at $45^{\circ} \mathrm{C}$. Later Boncompain et al (1986) in their analysis of the same bearing, in addition, approximated the distorted bearing internal surface to be a 
circle and determined that the nominal radial clearance of $145 \mathrm{~mm}$ and eccentricity ratio of 0.8 at $20^{\circ} \mathrm{C}$ is equivalent to $157 \mathrm{~mm}$ and 0.74 respectively at $45^{\circ} \mathrm{C}$. Khonsari and Wang (1991) also performed analysis on this bearing, by including thermal expansion for both journal and bearing and elastic deformation of bearing and determined that the collective effect of these factors yielded satisfactory comparisons with the experimental data. Figure 4 is the comparison of temperature distribution at the bearing symmetry plane, predicted using 3 internal Lobatto points each across film and journal radius and 5 internal Lobatto points across bearing thickness with the predictions of Boncompain et al (1986). Obviously, the plots are not to scale, with the dimensions of the film region highly exaggerated, so that temperature variations within the film can be clearly seen. The general trend in the temperature contours predicted by both procedures is similar. Both analyses predict a peak temperature of about $52^{\circ} \mathrm{C}$. However, the present analysis predicts the maximum fluid temperature around the film rupture region, whereas, Boncompain et al predict this occurrence in the cavitated region. In the THD model, since heat is being dissipated to ambient through the bearing metal, the bearing inside surface is cooler than the fluid temperature in this region. Hence, the maximum temperature should occur in the vicinity of minimum film thickness, where the viscous dissipation is the largest. Notice that, the oil film near the supply groove is cooler than the bearing/journal metal temperature. In this case, much of the journal metal is at about $46^{\circ} \mathrm{C}$. The isotherms in the bearing metal are primarily in the radial direction.

We now compare predicted bearing temperatures with values determined in various experiments. Figures 5 and 6 compare the present predictions of pressure and temperature profile around the circumference of the bearing at the symmetry plane with the experimental data of Ferron et al (1983) when the bearing is operating at $2000 \mathrm{rpm}$ under $4 \mathrm{kN}$ load and at $4000 \mathrm{rpm}$ under $6 \mathrm{kN}$ load. The predicted pressure profile, considering only the differential dilatation, agrees very well with the data and the predicted bearing inner surface temperature variation is close to the experimental data, particularly in the cavitated region. Since, the active part of the thermocouples are located near the film-bearing interface, the fluid-metal interface temperatures are used for this comparison. As can be seen from Fig. 4, the temperature profile in the bearing is predominantly in the radial direction and temperature difference between inside and outside surfaces are only about $2-4^{\circ} \mathrm{C}$. Nondimensional side leakage flow rate $(\mathrm{Q} / \mathrm{LCU})$ predicted by the present procedure are compared with the experimental results of Ferron et al (1983) for a wide range of eccentricity ratios and at two different speeds (2000 $\mathrm{rpm}$ and $4000 \mathrm{rpm}$ ) in Fig. 7. The agreement is excellent. Also note that, for an isothermal analysis, irrespective of the speed, the nondimensional flow rate will be the same. The thermal effects on the flow rate in the bearing are clearly brought out in this comparison. Figure 7 also provides plots of power loss at these conditions. There is a significant increase in the power 
loss due to increased speed, but it does not vary much due to variation in eccentricity ratio or applied load.

Mitsui (1987) conducted a number of experiments on circular journal bearings. The bearing with an $L / D$ value of 0.7 , had one axial groove of $10^{\circ}$ arc, extending $85 \%$ of length, located at the crown of top pad. Figure 8 compares the predictions of bearing outside temperature with the experiments conducted at $3.73 \mathrm{kN}$ and $1750 \mathrm{rpm}$, with two different lubricants, transformer oil and \#140 turbine oil. The predicted temperature variation is close to the experimental data, particularly in the cavitated region. The higher the viscosity of lubricant used, the larger the temperature rise. Though the viscosity of turbine oil is more than six times that of transformer oil at supply temperature, the effective viscosity in the film is not that much different, due to the larger temperature rise.

Lund and Tonnesen (1984) conducted experiments on a two axial groove journal bearing, each groove extending $10^{\circ}$ circumferentially and half the length axially. Figure 9 compares the circumferential profile of the bearing wall temperature at the bearing symmetry plane, at the same applied load was $5.6 \mathrm{kN}$ and when the journal speed $3500 \mathrm{rpm}$ and 5000 rpm. The predicted temperature variation is in close agreement with the experimental data, except upstream of the second groove. Fitzgerald and Neal (1992) also conducted experiments on two axial groove journal bearings, each groove extending $28.7^{\circ}$ circumferentially and $80 \%$ of the bearing length. Figure 10 provides a comparison of the circumferential profile of the bearing wall temperature at the bearing symmetry plane, when the applied load was $9.43 \mathrm{kN}$ at the journal speed of $8000 \mathrm{rpm}$; but for different $\mathrm{L} / \mathrm{D}$ ratios of 0.5 and 1.0. Again, the predicted temperature variation is in close agreement with the experimental data, except upstream of the second groove. In the twin axial groove bearings, although there is no significant supply of lubricant through the second groove, the groove is assumed to be flooded. Hence, at the upstream of the second groove the striation width is comparatively larger. Since, the heat transferred to bearing metal in the cavitated region is at the striation surface, more heat is dissipated to the ambient at this region. Therefore, the predicted metal temperature in this region rises a bit before dropping. In the experiment, there could be mixing of hot oil with the cooler lubricant causing the fluid temperature to reduce at the second groove, as can be seen in Fig. 9. Considering this, the rise in temperature of the fluid at the upstream of second groove is compatible with the predictions. Such a situation is, of course, difficult to treat analytically. Knight and Niewiarowski (1990) also predicted the temperature profile for the above cases using a gas bubble model and obtained good agreement with the experimental data in the cavitated region. The gas bubble model assumes that the film is continuous in the cavitated region, but the effective density and viscosity of the film are varied according to the gas fraction. In the present approach, the film occupies only a part of the volume in the cavitated 
region, corresponding to the mass fraction and only the film is considered for thermal analysis. Ma and Taylor (1994) developed a seperation cavitation model to account for the oil back flow from the groove into the cavitated region and were able to determine the temperature fade. This present model also, by conserving mass flow, does take into account back flow in the groove and correctly determines the temperature fade in the cavitated region at the trailing groove.

In general, the present predictions compare very well with various analytical and experimental data. While comparing the predicted results with experimental data, it should be recognized that there will be number of variables in the experiment that can not be easily simulated theoretically. To name a few: operating clearance, film shape, mixing at the groove, heat transfer coefficient, heat dissipation to other components in the test rig etc. Considering all these factors, it can be said that the present procedure predicts the temperature profiles rather well.

\section{CONCLUSIONS}

An efficient numerical procedure to determine the thermohydrodynamic performance of the cavitating bearings is developed considering mass and energy conservations and more accurately treating the cavitated region and its boundaries. This procedure requires few points across the film thickness strategically located and the profile is expressed using collocated polynomials. The details of the theoretical and numerical formulations are presented. The predictions using this procedure are compared with various numerical and experimental data, and lead us to the following major conclusions:

(i) The predictions are in good agreement with the experimental data and predictions by others.

(ii) With just 3 internal points across the film (and across the bearing shell and journal radius), the temperature profile can be well predicted.

(iii) Although some matrix operations are required with this procedure, the computer time requirement is very small.

(iv) The approach gives a logical treatment of mass and energy conservation across the cavitation boundaries and determines three dimensional viscosity, velocity, flow, viscous dissipation and temperature profiles.

(v) With a dual time step procedure, the temperature distributions across the film and the metals are simultaneously determined, resulting in faster steady state solutions. 


\section{ACKNOWLEDGMENT}

This work was performed under NASA Grant NCC 3-291. The author acknowledges with pleasure the assistance and encouragement of David E. Brewe, who monitored support by the U.S. Army Vehicle Propulsion Directorate at the NASA/Lewis Research Center, Cleveland, Ohio. The author profoundly thanks Prof. Harold G. Elrod for his guidance, suggestions and comments throughout the duration of this work.

\section{REFERENCES}

Abramovitz, M. and Stegun, I.A., 1965, Handbook of Mathematic Functions with Formulas, Graphs and Mathematical Tables, U. S. Bureau of Standards, p-920. Anderson, D.A., Tennehill, J.C. and Pletcher, R. H., 1984, Computational Fluid Mechanics and Heat Transfer, Hemisphere Publishing Corporation, New York, pp 99-117. Boncompain, R., Fillon, M. and Frene, J., 1986, "Analysis of Thermal Effects in Hydrodynamic Bearings", ASME Journal of Tribology, Vol 108, pp 219-224.

Brewe, D.E., 1986, "Theoretical Modeling of Vapor Cavitation in Dynamically Loaded Journal Bearings", ASME Journal of Tribology, Vol 108, pp 628-638.

Douglas, J. and Gunn, J.E., 1964, "A General Formulation of Alternating Direction Methods Part I: Parabolic and Hyperbolic Problems", Numerische Mathematik, Vol 6, pp 428-453.

Dowson, D., Hudson, J., Hunter, B. and March, C., 1966-67, "An Experimental Investigation of the Thermal Equilibrium of Steadily Loaded Journal Bearings", Proc. Inst. of Mech. Engr, Vol 101, Part 3B, pp 70-80.

Elrod, H.G. and Adams, M.L., 1974, "A Computer Program for Cavitation and Starvation Problems', Cavitation and Related Phenomena in Lubrication, Mechanical Engineering Publications, New York, pp 37-41.

Elrod, H.G., 1981, "A Cavitation Algorithm", ASME Journal of Lubrication Technology, Vol 103, pp 350-354.

Elrod, H.G., 1991, "Efficient Numerical Method for Computation of Thermohydrodynamics of Laminar Lubricating Films", ASME Journal of Tribology, Vol 113, pp 506-511.

Elrod, H.G. and Vijayaraghavan, D., 1995, "Film Temperatures in the Presence of Cavitation", NASA CR - 195481; US Army ARL - CR - 231. 
Ferron, J., Frene, J. and Boncompain, R., 1983, "A Study of the Thermohydrodynamic Performance of a Plain Journal Bearing; Comparison between Theory and Experiments", ASME Journal of Lubrication Technology, Vol 105, pp 422-428.

Fitzgerald, M.K. and Neal, P.B., 1992, "Temperature Distribution and Heat transfer in Journal Bearings", ASME Journal of Tribology, Vol 114, pp 122-130.

Han, T. and Paranjpe, R.S., 1990, "A Finite Volume Analysis of Thermohydrodynamic Performance of Finite Journal Bearings", ASME Journal of Tribology, Vol 112, pp 557-566.

Heshmat, H. and Pinkus, O., 1986, "Mixing Inlet Temperatures in Hydrodynamic Bearings", ASME Journal of Tribology, Vol 108, pp 221-248.

Jakobsson, B. and Floberg, L., 1957, "The Finite Journal Bearing Considering Vaporisation", Transactions of Chalmers University of Technology, Report No. 190.

Khonsari, M.M. and Beaman, J.J., 1985, "Thermohydrodynamic Analysis of Laminar Incompressible Journal Bearings", ASLE Transactions, Vol 29, pp 141-150.

Khonsari, M. M., 1987, "A Review of Thermal Effects in Hydrodynamic Bearings, Part I: Slider and Thrust Bearings; Part II: Journal Bearings", ASLE Transactions, Vol 30, pp 19-33.

Knonsari, M. M. and Wang, S. H., 1991, "On the Fluid-Solid Interaction in Reference to Thermohydrodynamic Analysis of Journal Bearings", ASME Journal of Tribology, Vol 113, pp 398-404.

Knight, J.D. and Niewiarowski, A.J., 1990, "Effects of Two Film Rupture Models on the Thermal Analysis of a Joumal Bearing", ASME Journal of Tribology, Vol 112, pp 183-188.

Lund, J. W. and Tonnesen J, 1984, "Approximate Analysis of Temperature Conditions in a Journal Bearing Part II: Applications", ASME Journal of Tribology, Vol 106, pp 237-245.

Ma , M. T. and Taylor, C. M., 1994, "Prediction of Temperature Fade in the Cavitation Region of Two-Lobe Journal bearing", Proc. Inst. of Mech. Engr, Part J: Journal of Eng. Tribology, Vol 208, pp 133-139.

Mitsui, J., Hori, Y. and Tanaka, M., 1983, "Thermohydrodynamic Analysis of Cooling Effect of Supply Oil in Circular Journal Bearing", ASME Journal of Lubrication Technology, Vol 105, pp 414-421.

Mitsui, J., 1987, "A Study of Thermohydrodynamic Lubrication in a Circular Journal Bearing", Tribology International, Vol 20, No. 6, pp 331-341. 
Mittwollen, N. and Glienicke, J., 1990, "Operating Conditions of Multi-Lobe Journal bearings Under High Thermal Loads", ASME Journal of Tribology, Vol 1112, pp 330-340.

Olsson, K.O., 1965, "Cavitation in Dynamically Loaded Bearings", Transactions of Chalmers University of Technology, Report No. 308.

Ott, H. H. and Paradissiadis, G., 1988, "Analysis of Thermal Effects in Hydrodynamic Bearings", ASME Journal of Tribology, Vol 110, pp 439-447.

Paranjpe, R. S., 1992, "Analysis of Non-Newtonian Effects in Dynamically Loaded Journal bearings Including Mass Conserving Cavitation", ASME Journal of Tribology, Vol 114, pp 736-746.

Paranjpe R. S. and Han T., 1994a, "A Study of the Thermohydrodynamic Performance of Steadily Loaded Journal Bearings", STLE Tribology Transactions, Vol 37, pp 679-690.

Paranjpe R. S. and Han T., 1994b, "A Transient Thermohydrodynamic Analysis Including Mass Conserving Cavitation for Dynamically Loaded Journal Bearings", Presented at ASME/STLE Tribology Conference, Maui, Hawaii, Paper: 94-Trib-36.

Pinkus, O., 1990, Thermal Effects of Fluid Film Tribology, ASME Press, New York. Villadsen, J. and Michelsen, M.L., 1978, Solution of Differential Equation Models by Polynomial Approximations, Prentice-Hall, Englewood Cliffs, New Jersy. 

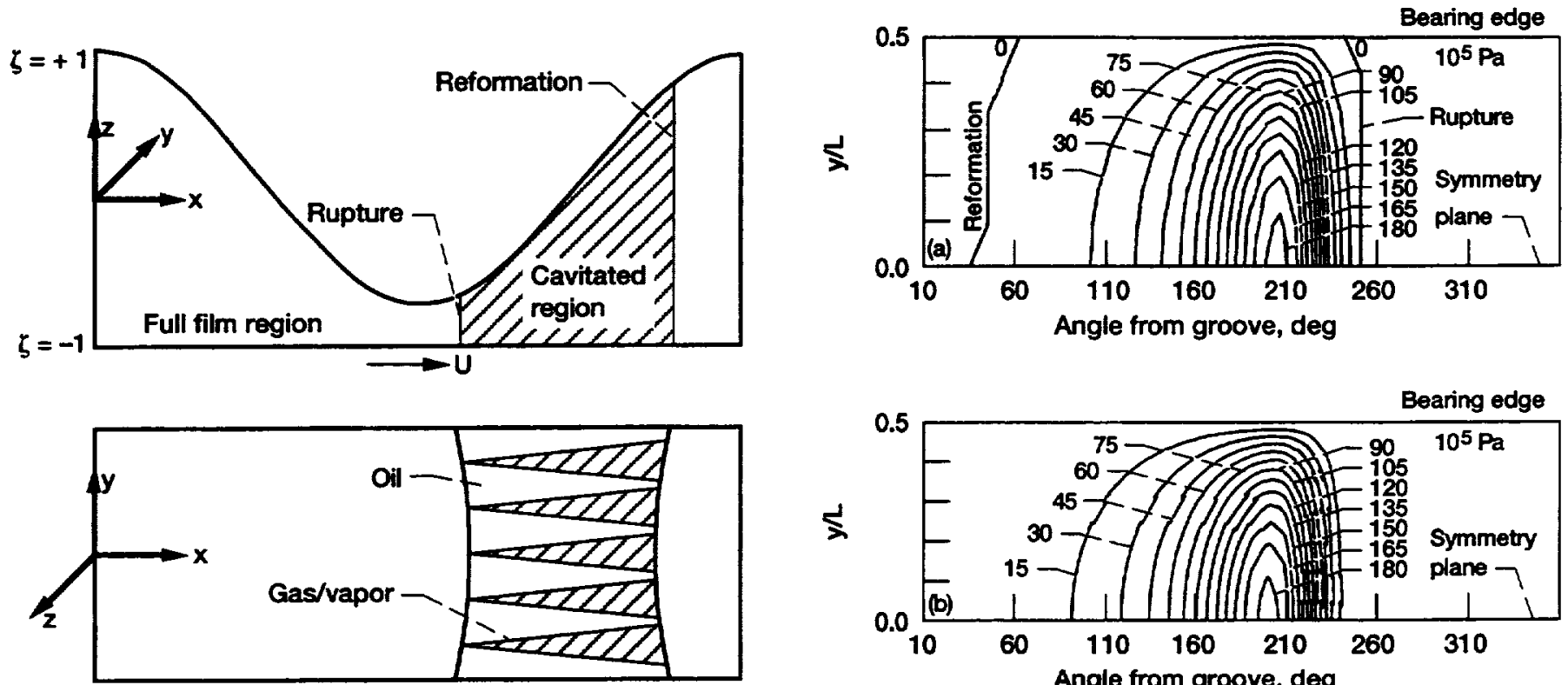

Figure 1.-Schematic diagram of a cavitating bearing.

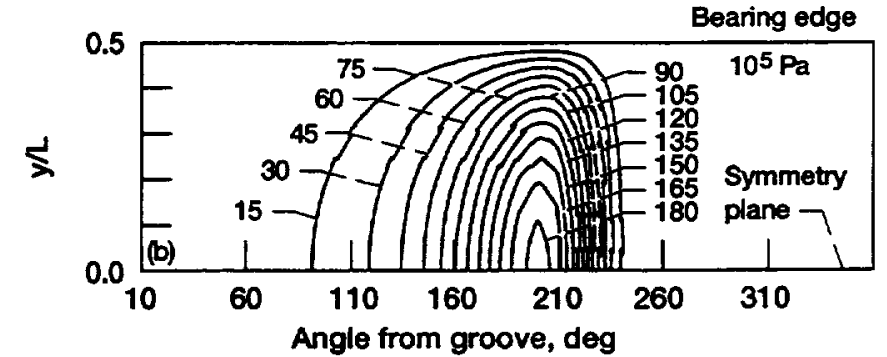

Figure 2.-Comparison of pressure contours at symmetry plane. (a) Present. (b) Han and Paranjpe, 1990.
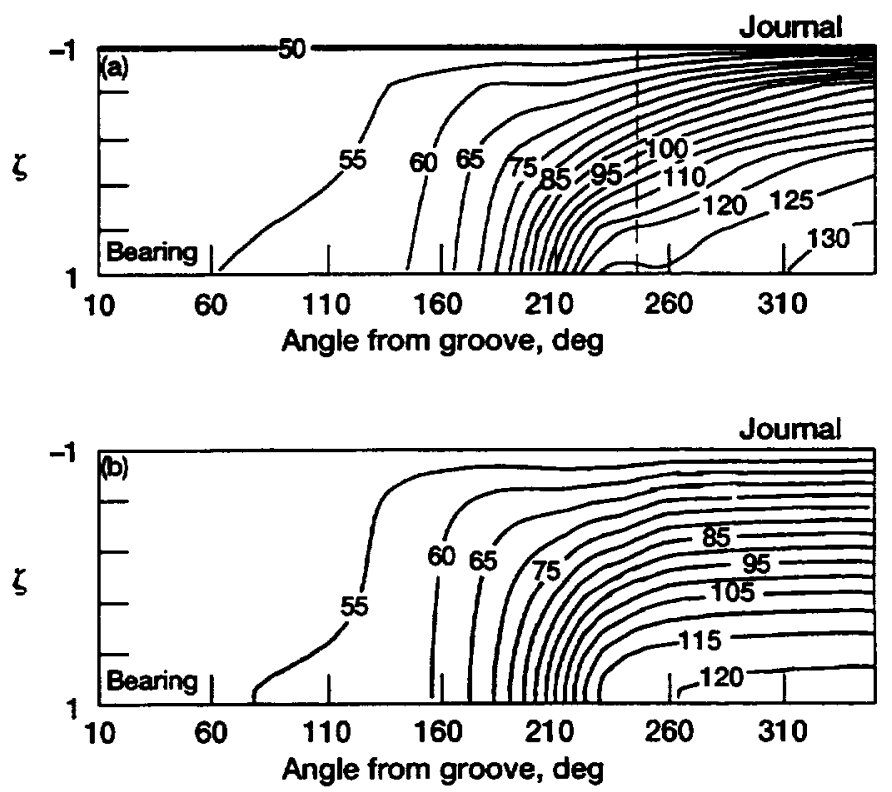

Figure 3.-Comparison of temperature contours at symmetry plane. (a) Present. (b) Han and Paranjpe, 1990. 


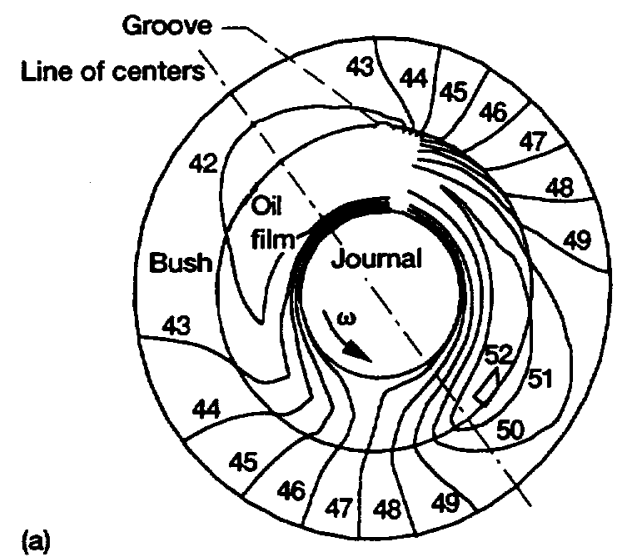

(a)

(b)

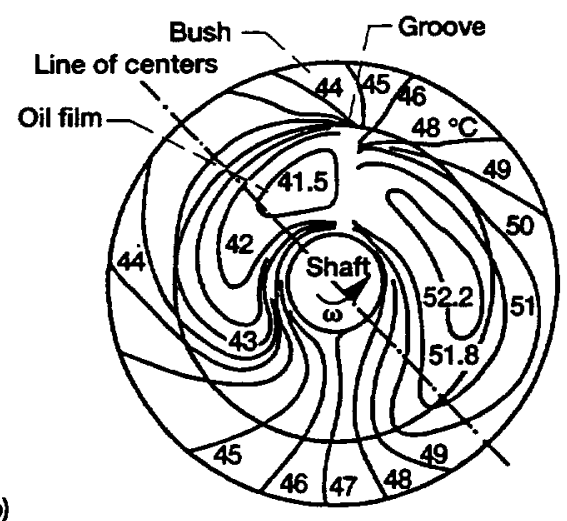

Figure 4.-Comparison of temperature contours at symmetry plane. (a) Present. (b) Boncompain et al., 1986.

\section{Present Expt}

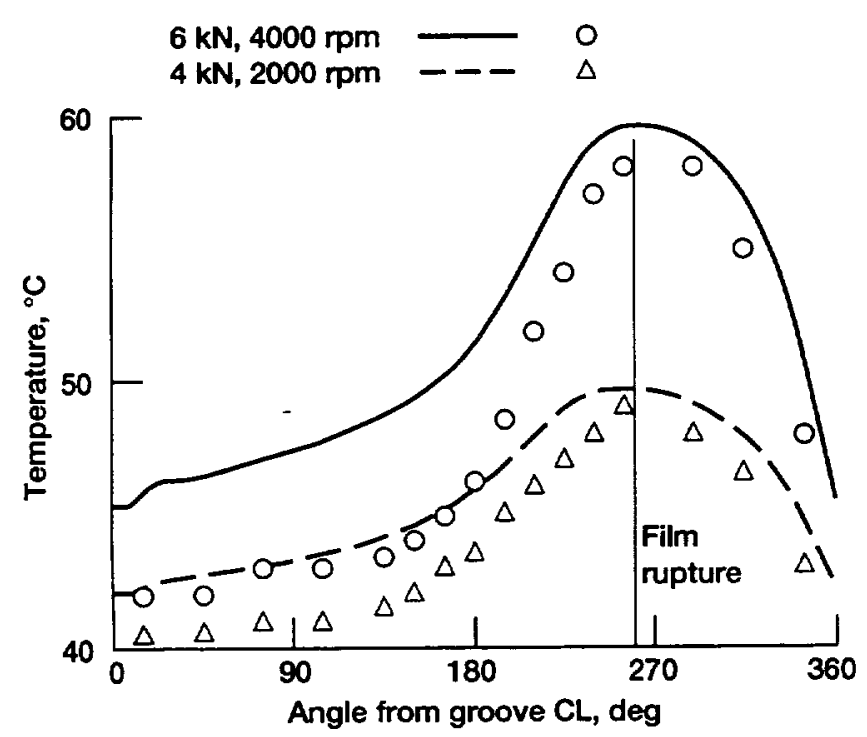

Figure 6.-Comparison of bearing inside surface temperature variation at symmetry plane (Ferron et al., 1983).

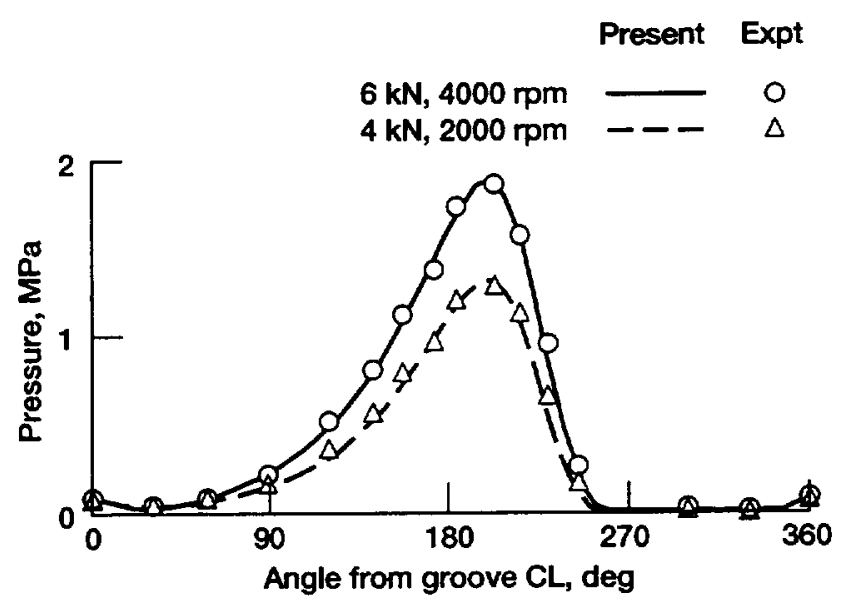

Figure 5.-Comparison of pressure variation at symmetry plane (Ferron et al., 1983).

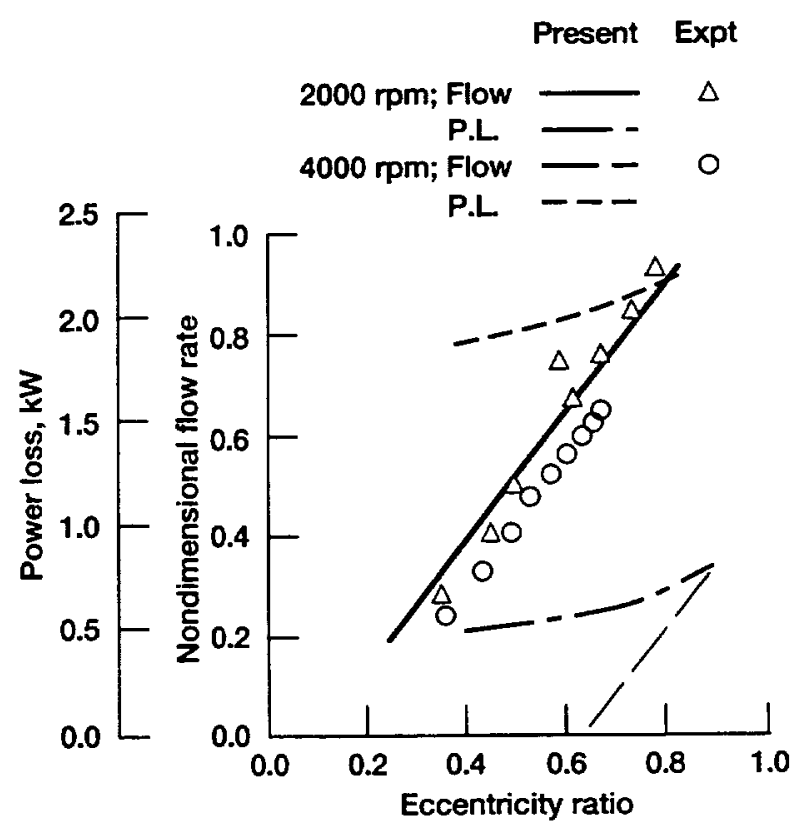

Figure 7.-Comparison of flow rate and power loss (Ferron et al., 1983). 


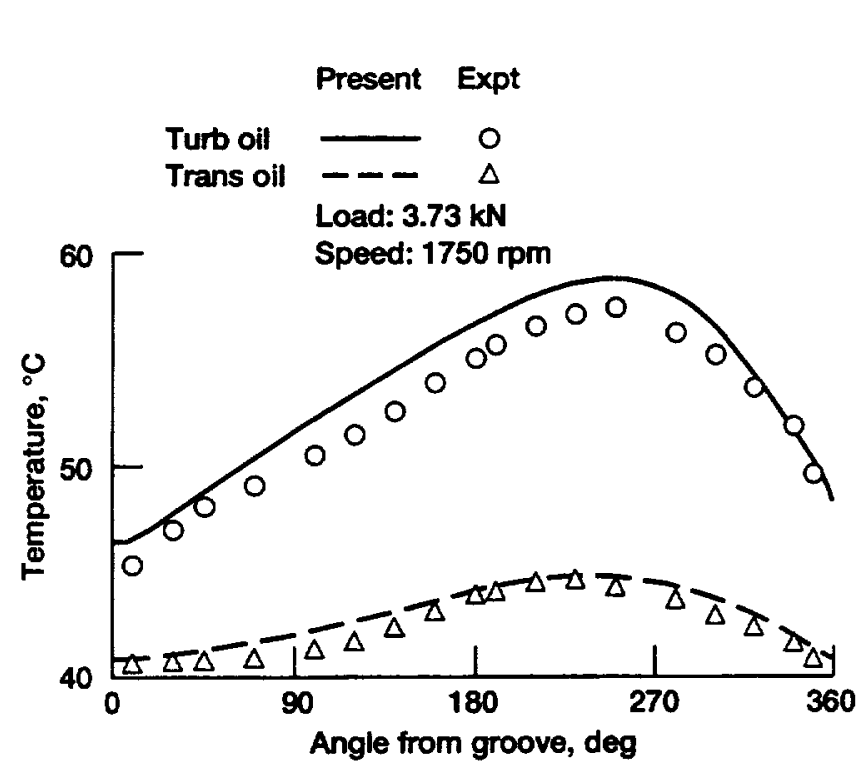

Figure 8.-Comparison of bearing outside temperature at symmetry plane (Mitsui, 1987).

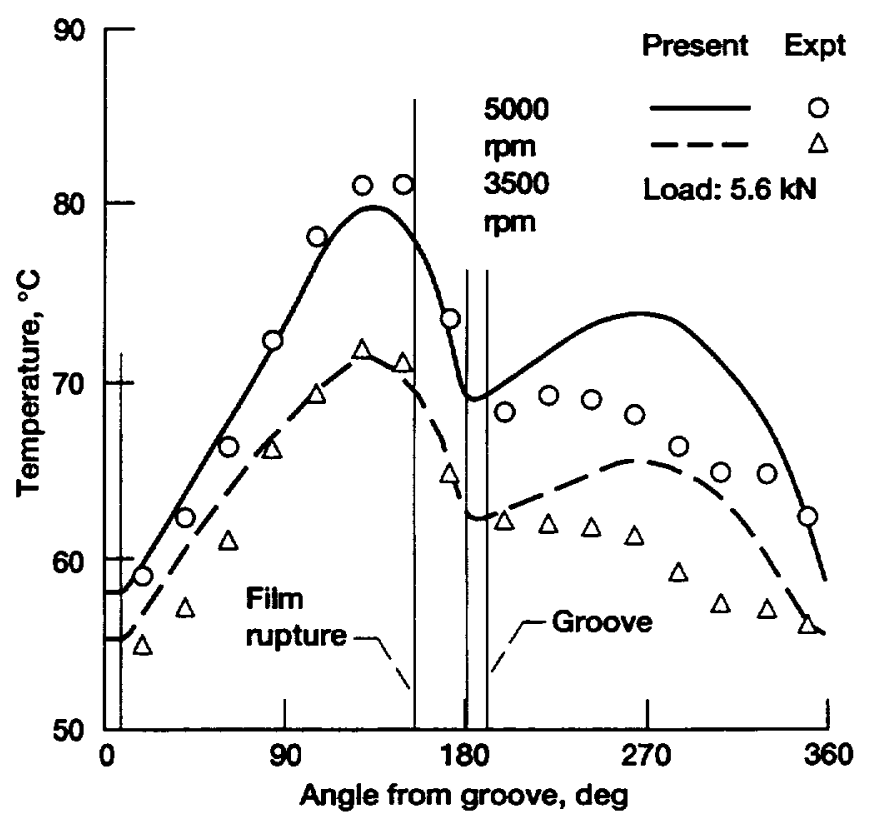

Figure 9.-Comparison of bearing outside temperature at symmetry plane (Lund and Tonnesen, 1984).

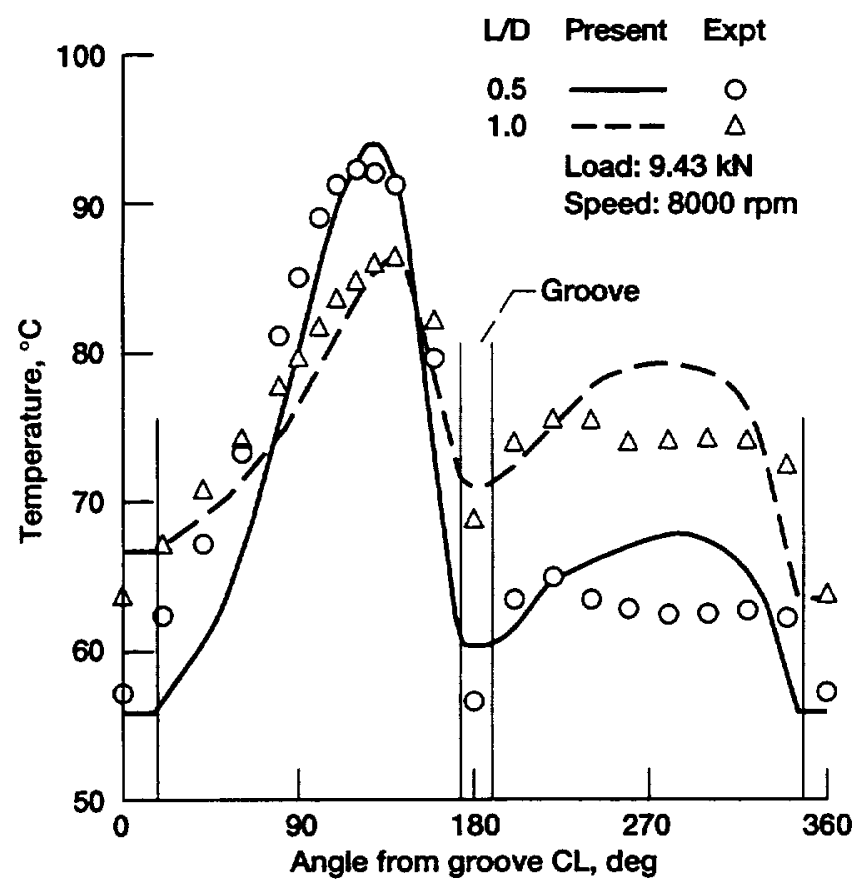

Figure 10.-Comparison of bearing outside temperature at symmetry plane (Fitzgerald and Neal, 1992). 


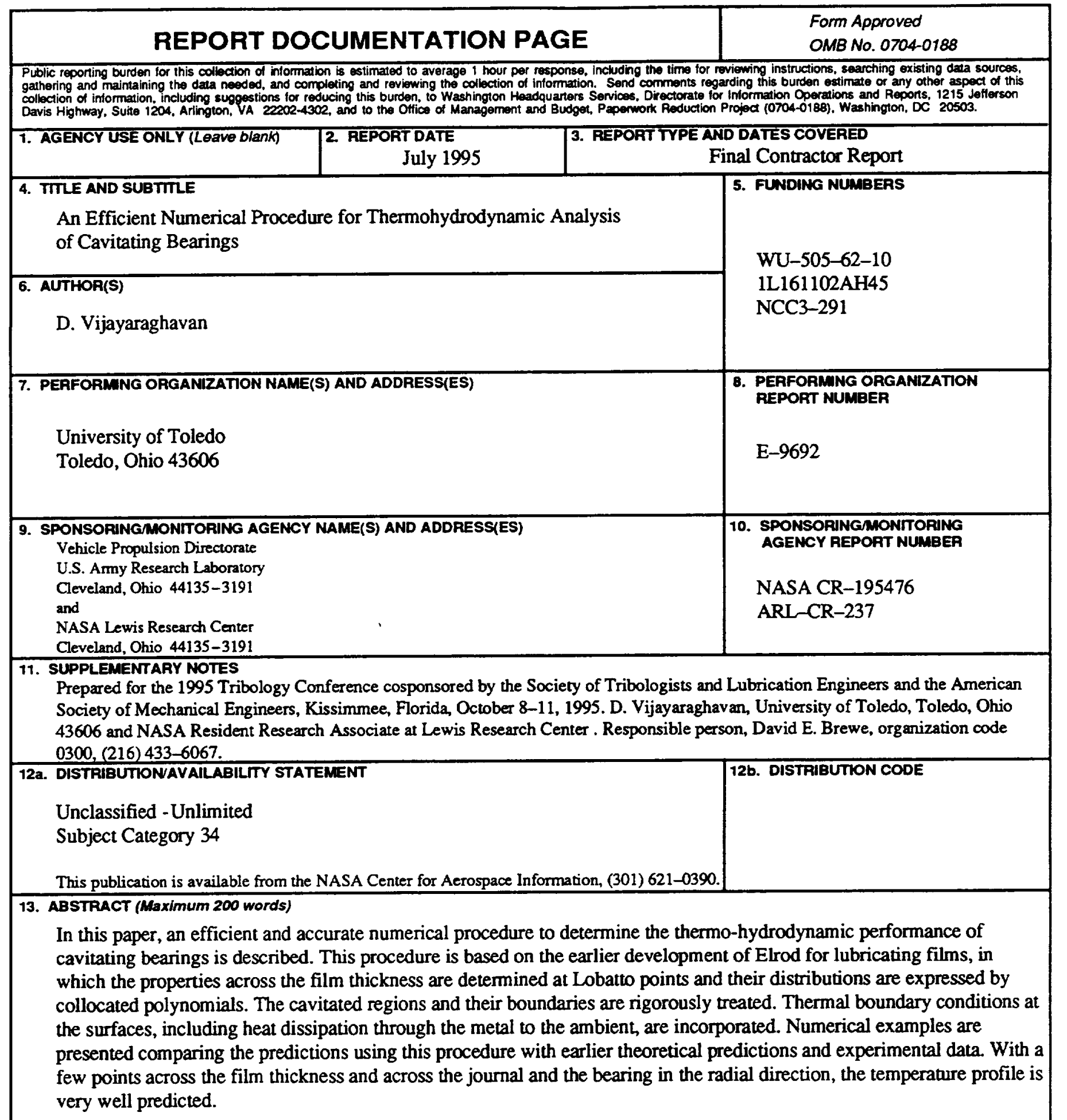


